\title{
SSR Marker Based Molecular Characterisation of Finger Millet Accessions of India and Anuradhapura District of Sri Lanka
}

\author{
P.W. Wakista ${ }^{1 *}$, P.N. Dasanayaka ${ }^{2}$, R.J. Illeperuma ${ }^{1}$, S.A.C.N. Perera ${ }^{3}$ \\ ${ }^{1}$ Genetech, No 54, Kitulwatta Road, Colombo 08, Sri Lanka \\ ${ }^{2}$ Department of Botany, University of Sri Jayewardenepura, Sri Lanka \\ ${ }^{3}$ Genetics and Plant Breeding Division, Coconut Research Institute, Lunuwila, Sri Lanka \\ *paro.wee@gmail.com
}

Molecular characterisation of germplasm accessions gives precise information about the extent of genetic diversity which helps in the development of appropriate breeding and conservation programs. Although most of the crops has gained much progress with the advancement of molecular marker technology, Finger millet is still in its initial stage of improvement and it requires to be focused in large scale since Finger millet is more nutritious than most of the other cereal grains.

This study was conducted to reveal the genetic diversity of Finger millet accessions of India and 15 accessions of Anuradhapura district of Sri Lanka using ten SSR (Simple Sequence Repeats) markers (UGEP 05, 10, 12, 15, 24, 68, 81, 102, 106 and 110).

Genetic distances between Finger millet accessions were explored using frequency based shared allele distance matrix. The highest distance of 0.9000 was observed between accession No.000924 (India) and 000926 (India), 000962 (India), 000964 (India), 001766 (Anuradhapura), 007613 (Anuradhapura). Accession No: 000925 (India) and 000927 (India); 005047 (Anuradhapura) and 006572 (Anuradhapura); 007072 (Anuradhapura), 001201 (Anuradhapura) and 007071 (Anuradhapura); 001766 (Anuradhapura) and 007770 (Anuradhapura); 000964 (India) and 007614 (Anuradhapura) showed significant genetic similarity. Dendrogram constructed on the basis of SSR polymorphism contained two main clusters leaving five of the Indian accessions as an out group. One main cluster comprised entirely of four accessions of India while the other having accessions of Anuradhapura with seven remaining Indian accessions. Comparatively higher genetic diversity was observed within Indian accessions than that of Anuradhapura district. Clustering of seven Indian accessions with that of Anuradhapura district implies their genetic relatedness irrespective to their geographical origin. Genetically distinct individuals identified within Indian accessions and between Indian and Anuradhapura accessions from the study can be considered as potential candidates in crop improvement programs.

Keywords: Finger millet, SSR, Genetic diversity 